myoclonus confirmed that EMG activity was preceded by EEG activity by $8-15 \mathrm{~ms}$, recorded over the contralateral rolandic area, suggesting a cortical origin. (Guerrini R, Bonanni P, Patrignani A et al. Autosomal dominant cortical myoclonus and epilepsy (ADCME) with complex partial and generalized seizures. A newly recognized epilepsy syndrome with linkage to chromosome 2p11.1-q12.2. Brain December 2001;124:2459-2475). (Respond: Professor R Guerrini, Neurosciences Unit, Institute of Child Health and Great Ormond Street Hospital for Children, The Wolfson Center, Mecklenburgh Square, London WC1N 2AP).

COMMENT. This autosomal dominant cortical myoclonus epilepsy syndrome (ADCME) in an Italian pedigree shares some of the characteristics of familial adult myoclonic epilepsy (FAME) described in pedigrees from Japan, except that FAME is linked to chromosome 8q23.3-q24.1, whereas ADCME linkage is to chromosome 2p11.1-q12.2. Clinical characteristics of ADCME include mainly adult onset, nonprogressive course, rhythmic myoclonus enhanced during movement, generalized tonic-clonic seizures, and sometimes complex partial seizures.

\title{
OUTCOME OF REFRACTORY STATUS EPILEPTICUS
}

The records of twenty-two children, ages 4.5 months to 18 years, treated for refractory status epilepticus (RSE) between 1992 and 2000, were reviewed retrospectively at Children's Hospital, Boston. Treatment consisted of high-dose anesthetic agents, including pentobarbital, midazolam, propofol, phenobarbital, alone or in combination, for periods ranging from 2 to 146 days (mean 31 days). Outcome correlated with duration of treatment: of 8 treated for $<7$ days, only 2 died and 4 returned to baseline neurologic state, whereas of 7 requiring treatment of RES for $>31$ days, 4 died and only 1 returned to baseline. A total mortality of $32 \%$ ( 7 cases) was related to etiology, age, and EEG. The most frequent etiology was presumed or known viral encephalitis affecting 10 patients, of whom 4 died. Of 4 children $<3$ years of age, none returned to baseline, 3 died, and one had new neurologic deficits after RSE. Of 6 children older than 10 years, 4 returned to baseline, and only one died. Mortality was $25 \%$ in 12 children with focal EEG abnormalities at onset of SE, and $40 \%$ in 10 with multifocal or generalized EEG abnormalities. No deaths occurred in 5 seizure susceptible children with remote symptomatic seizures not due to an acute provocation; their diagnoses were mental retardation, Rett's syndrome, old intracranial hemorrhage, atypical Rassmussen's encephalitis, and developmental delay. (Sahin M, Menache CC, Holmes GL, Riviello JJ Jr. Outcome of severe refractory status epilepticus in children. Epilepsia Nov 2001;42:1461-1467). (Reprints: Dr M Sahin, Children's Hospital, 300 Longwood Ave, Enders 250, Boston, MA 02115).

COMMENT. Refractory status epilepticus in childhood carries a high mortality (32\%) and morbidity that are related to the etiology, age, EEG, and duration of suppressive treatment. Younger patients with presumed encephalitis and multifocal EEGs require prolonged suppressive therapy and the outcome is especially poor.

The findings were similar in a study of 346 adolescent and adult patients with status epilepticus reported from the Netherlands (Scholtes FB et al. Epilepsia 1995;35:1104-1112). A poor outcome was related especially to the underlying cause and status symptomatic of an acute illness, a duration of $>4$ hours, the occurrence of medical complications, and inadequate therapy. Of 38 patients who died, $44 \%$ had received insufficient therapy.

A study in 74 adults with 83 episodes of status epilepticus at the Neurologial Institute, New York, found a mortality of $21 \%$ which was correlated with increased 
age and acute symptomatic seizures. Increased length of hospitalization and acute symptomatic seizures were predictors of functional disability in $23 \%$ of patients with nonfatal episodes. (Claassen J, Lokin JK, Fitzsimmons BFM et al. Predictors of functional disability and mortality after status epilepticus. Neurology January (1 of 2) 2002;58:139-142).

Regarding long-term outcome, the occurrence of status with recovery had no adverse effects in a cohort of 342 children, when followed prospectively for a mean of 72 months (Shinnar S et al. Dev Med Child Neurol 1995 (suppl 72);37:116 (abstract)). It appears that mortality with status is related particularly to the cause of the status and the association of an acute illness such as encephalitis or encephalopathy, and sometimes to inadequate therapy. Although the majority of single, generalized tonic-clonic seizures are self-limiting and no more than 2 to 4 minutes duration, a seizure lasting 5 minutes or more may progress to status and should be treated aggressively. The prevention of status and the effective treatment of status are dependent on the avoidance of delay in initiating anticonvulsant therapy, and the adherence to a well-defined protocol outlined for emergency room physicians to follow. (Leppik IE, 2000; see Progress in Pediatric Neurology III, PNB Publ, 1997;102).

\section{VIDEO-EEG IN DIAGNOSIS OF EPILEPSY IN RETARDED CHILDREN}

Video-EEG monitoring was used to distinguish epileptic and non-epileptic events in 193 children, mean age 9.6 years, who presented with paroxysmal symptoms of uncertain etiology at Children's Hospital, Los Angeles, CA. Diagnosis was established in 130 (67\%). Seventy (36\%) were mentally retarded (MR). Epileptic seizures were identified in 67 (51\%), non-epileptic events in $54(41 \%)$, and both epileptic and non-epileptic events in $9(7 \%)$ children. Children with MR had predominantly epileptic seizures on long studies and non-epileptic events on short studies. A diagnosis was established in $82 \%$ following long studies and in $62 \%$ with short studies. The most common seizure type was complex partial, and non-epileptic symptoms were behavioral, psychogenic, and physiological events. Children with MR were more likely than children without MR to have events during the studies, but the frequencies of epileptic and non-epileptic events were similar in the two groups. Management was modified following diagnostic studies. (Thirumalai S, Abou-Khalil B, Fakhoury T, Suresh G. Video-EEG in the diagnosis of paroxysmal events in children with mental retardation and in children with normal intelligence. Dev Med Child Neurol Nov 2001;43:731-734). (Respond: Dr Shanti Thirumalai, Children's Hospital, 4650 Sunset Blvd, Mail Stop 82, Los Angeles, CA 90027).

COMMENT. Improved diagnosis and more appropriate management following video-EEG in children with paroxysmal events should prompt more general use of this test, especially in children with mental retardation.

Eight infants with early-infantile epileptic encephalopathy (Ohtahara syndrome) had seizures that correlated with the ictal burst of the suppressionburst pattern recorded by video-EEG, in a report from the Bambino Gesu' Children's Hospital, Rome, Italy (Fusco L et al. Brain Dev 2001;23:708-714).

\section{CARNITINE LEVEL CHANGES WITH THE KETOGENIC DIET}

The effects of the ketogenic diet (KD) on carnitine levels were determined in 38 consecutive patients with epilepsy treated at Rush-Presbyterian-St Luke's Medical Center, Chicago, IL. Carnitine levels were determined at $0,1,6,12$, and 24 months of diet treatment. Reduced total plasma carnitine (TC) at diet initiation was related to multiple antiepileptic drugs (AED), but only 3 had TC deficiency (range, 